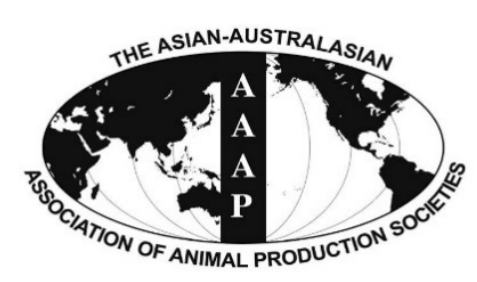

\begin{tabular}{c} 
Open Access \\
$\begin{array}{c}\text { Asian Australas. J. Anim. Sci. } \\
\text { Vol. 29, No. 4 : 549-554 April } 2016 \\
\text { http://dx.doi.org/10.5713/ajas.15.0426 }\end{array}$ \\
\hline www.ajas.info \\
pISSN 101 1-2367 eISSN 1976-5517
\end{tabular}

\title{
Growth Performance, Relative Meat and Organ Weights, Cecal Microflora, and Blood Characteristics in Broiler Chickens Fed Diets Containing Different Nutrient Density with or without Essential Oils
}

\author{
Sang-Jin Kim ${ }^{1,2, a}$, Kyung-Woo Lee ${ }^{1, a}$, Chang-Won Kang ${ }^{1}$, and Byoung-Ki An ${ }^{1, *}$ \\ ${ }^{1}$ Laboratory of Poultry Science, Department of Animal Science and Technology, \\ College of Animal Bioscience and Technology, Konkuk University, Seoul 05029, Korea
}

\begin{abstract}
The present study was conducted to investigate whether dietary essential oils could affect growth performance, relative organ weights, cecal microflora, immune responses and blood profiles of broiler chickens fed on diets containing different nutrient densities. A total of eight hundred-forty 1-d-old male broiler chicks were randomly allotted into twenty-eight pens (7 pens per treatment, 30 chicks per pen). There were four experimental diets containing two different nutrient densities and supplemented with or without essential oils. Experimental period lasted for 35 days. No clear interaction between nutrient density and essential oils on any of growth performance-related parameters was observed. Live body weights were affected $(p<0.05)$ by nutrient density at 21 days and by dietary essential oils at 35 days. Essential oils significantly $(p<0.05)$ increased daily body weight gain and feed conversion ratio during the periods of 22 to 35 and 1 to 35 days, but failed to affect feed intake during the entire experimental period. Daily weight gain at 1 to 21 days and feed intake at 1 to 21 and 1 to 35 days were significantly impaired $(p<0.05)$ by nutrient density. There were significant treatment interactions $(\mathrm{p}<0.05)$ on relative weights of bursa of Fabricius and abdominal fat contents. Finally, either essential oil or nutrient density did not influence the relative percentages of breast and leg meats, the population of cecal microflora, blood parameters and antibody titers against Newcastle disease and infectious bronchitis in broiler chickens. It was concluded that dietary essential oils, independent to nutrient density, failed to stimulate feed intake, but increased growth performance in broiler chickens. (Key Words: Growth Performance, Nutrient Density, Essential Oils, Broiler Chickens)
\end{abstract}

\section{INTRODUCTION}

Essential oils (EO) derived from spices and herbs, as single components or as mixed preparations, can play significant roles in supporting both performance and health status of poultry (Huyghebaert et al., 2011; Lee et al., 2011; Khattak et al., 2014). Beneficial effects of active plant substances in poultry nutrition include the stimulation of appetite and feed intake, the improvement of endogenous digestive enzyme secretion, the activation of immune response and antibacterial, antiviral, antioxidant and

\footnotetext{
* Corresponding Author: Byoung-Ki An. Tel: +82-2-450-3669, Fax:+82-2-453-3669, E-mail: abk7227@hanmail.net

${ }^{2}$ Yuhan Corp, Seoul 06927, Korea.

${ }^{a}$ These authors contributed equally to this work.

Submitted May 14, 2015; Revised Jul. 29, 2015; Accepted Aug. 24, 2015
}

antihelminthic actions (Lee et al., 2004; Jamroz et al., 2005; Zeng et al., 2015b). The aforementioned biological effects of EO could then lead to efficient nutrient utilization in poultry. Indeed, the supplementation of EO into low energy or nutrient density diet significantly increased daily weight gain and enhanced the digestibilities of nutrients compared with the low energy or nutrient density based control diet and exhibited comparable performance compared with a standard energy or nutrient density diet in pigs (Yan et al., 2010; Zeng et al., 2015a). In line with the latter studies, it was reported that dietary EO exhibited identical growth performance of broiler chickens fed the low-nutrient density (LND) diets compared with those fed the high-nutrient density (HND) diet (Scheuermann et al., 2009). Unfortunately, the absence of the LND control diet in the latter experiment made it difficult to prove the observed 
effect. Buchanan et al. (2008) reported that improvement of feed conversion ratio was only observed when EO was added into a HND vs LND diet. In contrast, no significant effect of EO on growth performance was observed in broiler chickens fed a threonine-deficient diet (Muhl and Liebert, 2007). In contrast to pig trials (Yan et al., 2010; Zeng et al., 2015a), the effect of EO on growth performance in broiler chickens fed a diet containing LND vs HND diet is inconclusive. Thus, the present experiment was intended to validate whether there is interaction between nutrient density and EO on growth performance in broiler chickens. In addition to production traits, various parameters such as blood metabolites, blood immune responses, organ weights, and cecal microflora were evaluated to see the presence or absence of the interaction between EO and nutrient density.

\section{MATERIALS AND METHODS}

\section{Experimental design}

The experimental facility was thoroughly cleaned and disinfected before the initiation of the experiment. A total of eight hundred-forty 1-d-old male broiler chicks (Ross 308) were obtained from local hatchery. Upon arrival, they were individually weighed and randomly placed into one of twenty-eight pens $(1.8 \mathrm{~m} \times 1.8 \mathrm{~m})$ with fresh rice husks as a bedding material and stocking density was set at $0.108 \mathrm{~m}^{2}$ per bird. The present experiment consisted of four dietary treatments which were given for 35 days (starter 1 to 21 days, finisher 22 to 35 days). Each treatment consisted of seven pens and each pen had 30 chickens ( $n=210$ chickens/treatment). There were four dietary experimental diets with two nutrient density diets (HND vs LND) supplemented with or without EO at $150 \mathrm{mg} / \mathrm{kg}$ of diet. The commercially available EO preparation (Biostrong 510, Delacon, Steyregg, Austria) consisted of a mixture of EO with thyme and star anise, Quillaja extracts, and bulking and anti-caking agents. Corn-soybean meal based HND and LND based diets were formulated (Table 1). The LND vs HND diet contained less energy and nutrients by one percent point of crude protein (CP), $50 \mathrm{kcal}$ of nitrogencorrected true metabolizable energy, 0.05 percent point of available phosphate, and 0.05 percent point of lysine. These nutrient reduction can be considered moderate as compared with the previous studies (Brickett et al., 2007; Zhao et al., 2009; Li et al., 2010; Mirshekar et al., 2013), in which the LND vs HND diet was formulated to reduce CP levels by 2 percent points, and energy levels by more than $150 \mathrm{kcal} / \mathrm{kg}$ of diet. Diet and water were provided ad libitum. Continuous lighting program was used and the temperature of facility was maintained at $32^{\circ} \mathrm{C}$ during the first week posthatch and gradually decreased to reach $25^{\circ} \mathrm{C}$ at 3 weeks and kept thereafter. At days 14 and 28, all broiler chicks used in this study were vaccinated against Newcastle
Table 1. Ingredient and chemical composition of the starter and finisher diets

\begin{tabular}{|c|c|c|c|c|}
\hline \multirow[t]{2}{*}{ Items } & \multicolumn{2}{|c|}{$\begin{array}{c}\text { Starter } \\
(1 \text { to } 21 \mathrm{~d})\end{array}$} & \multicolumn{2}{|c|}{$\begin{array}{l}\text { Finisher } \\
\text { (22 to } 35 \mathrm{~d})\end{array}$} \\
\hline & HND & LND & HND & LND \\
\hline \multicolumn{5}{|l|}{ Ingredients (\%) } \\
\hline Yellow corn & 55.12 & 59.83 & 59.92 & 63.78 \\
\hline Soybean meal & 33.23 & 30.97 & 29.22 & 27.18 \\
\hline Corn gluten meal & 3.36 & 2.89 & 2.95 & 2.99 \\
\hline Tallow & 4.48 & 2.57 & 4.35 & 2.59 \\
\hline DL-methionine (98\%) & 0.17 & 0.16 & 0.05 & 0.03 \\
\hline Salt & 0.33 & 0.33 & 0.33 & 0.33 \\
\hline Limestone & 1.19 & 1.38 & 1.56 & 1.74 \\
\hline Dicalcium phosphate & 1.64 & 1.38 & 1.12 & 0.86 \\
\hline Vitamin + mineral mixture ${ }^{1}$ & 0.40 & 0.40 & 0.40 & 0.40 \\
\hline Choline chloride $(50 \%)$ & 0.08 & 0.09 & 0.10 & 0.10 \\
\hline Total & 100.0 & 100.0 & 100.0 & 100.0 \\
\hline \multicolumn{5}{|l|}{ Calculated values } \\
\hline Crude protein $(\%)$ & 21.50 & 20.50 & 20.00 & 19.00 \\
\hline Crude fat $(\%)$ & 7.00 & 5.22 & 6.96 & 5.31 \\
\hline Crude fiber (\%) & 3.36 & 3.33 & 3.22 & 3.19 \\
\hline Crude ash (\%) & 6.00 & 5.84 & 5.66 & 5.51 \\
\hline $\mathrm{Ca}(\%)$ & 1.00 & 1.00 & 1.00 & 1.00 \\
\hline Available P (\%) & 0.40 & 0.35 & 0.30 & 0.25 \\
\hline Lysine (\%) & 1.13 & 1.07 & 1.02 & 0.97 \\
\hline Cys+met (\%) & 0.90 & 0.86 & 0.73 & 0.70 \\
\hline TMEn (kcal/kg) & 3,100 & 3,045 & 3,150 & 3,100 \\
\hline
\end{tabular}

HND, high-nutrient density; LND, low-nutrient density; TMEn, nitrogencorrected true metabolizable energy.

${ }^{1}$ Vit.+Min. mixture provided the following nutrients per $\mathrm{kg}$ of diet: vitamin $\mathrm{A}, 18,000 \mathrm{IU}$; vitamin $\mathrm{D}_{3}, 3,750 \mathrm{IU}$; vitamin $\mathrm{E}, 30 \mathrm{IU}$; vitamin $\mathrm{K}_{3}, 2.7 \mathrm{mg}$; vitamin $\mathrm{B}_{1}, 3.0 \mathrm{mg}$; vitamin $\mathrm{B}_{2}, 9.0 \mathrm{mg}$; vitamin $\mathrm{B}_{6}, 4.5 \mathrm{mg}$; vitamin $\mathrm{B}_{12}, 30.0 \mathrm{mg}$; niacin, $37.5 \mathrm{mg}$; pantothenic acid, $15 \mathrm{mg}$; folic acid, $1.5 \mathrm{mg}$; biotin, $0.07 \mathrm{mg}$; Fe, $75.0 \mathrm{mg}$; Zn, $97.5 \mathrm{mg}$; Mn, $97.5 \mathrm{mg}$; $\mathrm{Cu}, 7.5 \mathrm{mg} ; \mathrm{I}, 1.5 \mathrm{mg} ; \mathrm{Se}, 0.2 \mathrm{mg}$.

disease (ND) and infectious bronchitis (IB) by eye drop using the attenuated live mixed vaccine (Nobilis Ma5+Clone 30, MSD AH Korea Ltd., Seoul, Korea). All experimental protocols were approved by the Animal Care Committee of KonKuk University.

\section{Sampling}

Feed intake and body weight per pen were measured on a weekly basis and used to calculate feed conversion ratio. At 35 days, eight chickens per pen were randomly selected for blood sampling. Blood was collected from cardiac puncture immediately after cervical dislocation. Sera were obtained by gentle centrifugation (600 g for $15 \mathrm{~min}$ ) and stored at $-20^{\circ} \mathrm{C}$ prior to use. Immediately after blood sampling, organs such as liver, spleen, bursa of Fabricius and abdominal fat were excised, weighed and expressed as relative weight to live body weight. In addition, right breast and thigh meats were sampled and weighed. Finally, cecal contents were removed aseptically, placed into sterile tubes 
and kept on ice until used for gut microbiota analysis on the same day of the sampling.

Measurement of total cholesterol, glutamic-oxaloacetic transaminase and glutamic-pyruvic transaminase in serum samples

Blood parameters were measured as described elsewhere (Lee et al., 2010). In brief, total cholesterol concentration and activities of glutamic-oxaloacetic transaminase (GOT) and glutamic-pyruvic transaminase (GPT) in sera sampled at 35 days were measured according to the colorimetric method using cholesterol diagnostic kit (Cholesterol E kit, Asan Phamaceutical Co., Seoul, Korea) and GOT-GPT assay kits (Asan Phamaceutical Co., Korea).

\section{Measurement of infectious bronchitis- and Newcastle} disease-reactive antibody response

Viral antibodies against IB and ND in sera sampled at 35 days were determined (Lee et al., 2012) using commercial enzyme-linked immunosorbent assay kits (IDEXX Laboratory, Westbrook, ME, USA) according to the manufacturer's instructions. The results were expressed as antibody titer that was calculated using the formula provided by IDEXX.

\section{Enumeration of intestinal microflora}

Individual cecal contents were subjected to serial 10fold (w/v) dilution with ice-cold phosphate-buffered saline as suggested by Noh et al. (2014). Total microbes were counted after grown on total plate agar (Difco, Detroit, MI, USA), lactic acid bacteria on Man Rosa-Sharpe agar (Difco,
USA), and total coli forms on MacConkay agar (Difco, USA) at $37^{\circ} \mathrm{C}$ for $24 \mathrm{~h}$. Results obtained were presented as base-10 logarithm colony forming unit (cfu) per gram of cecal digesta.

\section{Statistical analysis}

Pen was considered an experimental unit. Two-way analysis of variance was performed to define the effect of diet (LND vs HND), EO $(0,150 \mathrm{mg} / \mathrm{kg}$ of diet $)$ and the diet by EO interaction on the variables using the general linear model procedure of SAS program (SAS, 2000). A statistical significance was preset at $\mathrm{p}<0.05$ unless otherwise stated.

\section{RESULTS}

There was no interaction between nutrient density and EO on the measured parameters except for bursa of Fabricius and abdominal fat in broiler chickens. Body weights were affected $(\mathrm{p}<0.05)$ by nutrient density at 21 days and by EO supplementation at 35 days. HND vs LND diet significant increased $(\mathrm{p}<0.05)$ daily body weight gain at 1 to 21 days (Table 2). Dietary EO significantly increased $(p<0.05)$ daily body weight gain during the periods of 22 to 35 and 1 to 35 days. Daily feed intake was not affected $(p>0.05)$ by dietary EO, but significantly reduced $(p<0.05)$ by the LND vs HND diet, especially during the periods of 1 to 21 and 1 to 35 days. Feed conversion ratio was significantly improved $(\mathrm{p}<0.05)$ by EO addition during the periods of 22 to 35 days and 1 to 35 days (Table 2). Either nutrient density or EO did not influence the relative weights of liver, spleen, breast and leg meat yields (Table 3), cecal

Table 2. Effect of dietary essential oils on growth performance in broiler chickens fed diets containing different nutrient densities ${ }^{1}$

\begin{tabular}{|c|c|c|c|c|c|c|c|c|}
\hline & \multirow{2}{*}{ HND } & \multirow{2}{*}{ LND } & \multirow{2}{*}{$\mathrm{HND}+\mathrm{EO}$} & \multirow{2}{*}{$\mathrm{LND}+\mathrm{EO}$} & \multirow{2}{*}{ Pooled SEM } & \multicolumn{3}{|c|}{ p-values } \\
\hline & & & & & & Diet & EO & Diet $\times \mathrm{EO}$ \\
\hline Initial BW (g/bird) & 40.3 & 40.3 & 40.3 & 40.3 & 0.033 & 0.880 & 0.880 & 0.880 \\
\hline 1 to $21 \mathrm{~d} \mathrm{BW}$ (g/bird) & 708.0 & 670.1 & 714.9 & 688.5 & 11.7 & 0.011 & 0.289 & 0.624 \\
\hline Final BW (g/bird) & $1,698.7$ & $1,656.4$ & $1,754.5$ & $1,708.9$ & 25.5 & 0.084 & 0.036 & 0.947 \\
\hline \multicolumn{9}{|l|}{ Feed intake (g/d/bird) } \\
\hline 1 to $21 \mathrm{~d}$ & 52.2 & 49.3 & 52.8 & 50.6 & 0.759 & 0.002 & 0.237 & 0.649 \\
\hline 22 to $35 \mathrm{~d}$ & 154.3 & 151.8 & 152.6 & 145.5 & 2.410 & 0.057 & 0.112 & 0.344 \\
\hline 1 to $35 \mathrm{~d}$ & 91.9 & 89.1 & 91.7 & 87.7 & 0.865 & 0.001 & 0.358 & 0.467 \\
\hline \multicolumn{9}{|l|}{ BW gain (g/d/bird) } \\
\hline 1 to $21 \mathrm{~d}$ & 32.5 & 30.0 & 32.1 & 30.2 & 0.644 & 0.002 & 0.866 & 0.668 \\
\hline 22 to $35 \mathrm{~d}$ & 71.3 & 70.5 & 74.3 & 73.2 & 1.321 & 0.472 & 0.042 & 0.925 \\
\hline 1 to $35 \mathrm{~d}$ & 48.0 & 47.5 & 50.4 & 48.8 & 0.732 & 0.162 & 0.023 & 0.440 \\
\hline \multicolumn{9}{|l|}{ Feed/gain (g/g) } \\
\hline 1 to $21 \mathrm{~d}$ & 1.61 & 1.64 & 1.64 & 1.68 & 0.018 & 0.064 & 0.064 & 0.784 \\
\hline 22 to $35 \mathrm{~d}$ & 2.17 & 2.16 & 2.06 & 1.99 & 0.058 & 0.496 & 0.024 & 0.609 \\
\hline 1 to $35 \mathrm{~d}$ & 1.92 & 1.88 & 1.82 & 1.80 & 0.029 & 0.307 & 0.005 & 0.731 \\
\hline
\end{tabular}

HND, high-nutrient density; LND, low-nutrient density; HND+EO, HND diet added with 150mg/kg of essential oils (EO); LND+EO, LND diet added with $150 \mathrm{mg} / \mathrm{kg}$ of EO; SEM, pooled standard error of the mean; BW, body weight.

${ }^{1}$ Values are expressed as means of seven replicates per dietary group. 
Table 3. Effect of dietary essential oils on relative organ and meat weights in broiler chickens fed diets containing different nutrient densities $^{1}$

\begin{tabular}{lcccccccc}
\hline & HND & \multirow{2}{*}{ LND } & \multirow{2}{*}{ HND+EO } & \multirow{2}{*}{ LND+EO } & \multirow{2}{*}{ Pooled SEM } & \multicolumn{3}{c}{$\mathrm{p}$-values } \\
\cline { 6 - 9 } & & & & & Diet & EO & Diet $\times$ EO \\
\hline Liver & 2.36 & 2.29 & 2.28 & 2.35 & 0.081 & 0.999 & 0.903 & 0.398 \\
Spleen & 0.12 & 0.14 & 0.13 & 0.13 & 0.010 & 0.327 & 0.999 & 0.327 \\
Bursa of Fabricius & 0.23 & 0.19 & 0.21 & 0.23 & 0.013 & 0.457 & 0.457 & 0.033 \\
Abdominal fat & 1.53 & 1.87 & 2.16 & 1.53 & 0.188 & 0.448 & 0.447 & 0.016 \\
Breast meat & 7.70 & 7.62 & 8.02 & 7.85 & 0.228 & 0.522 & 0.166 & 0.817 \\
Leg meat & 8.87 & 9.00 & 9.25 & 9.19 & 0.166 & 0.838 & 0.105 & 0.579 \\
\hline
\end{tabular}

HND, high-nutrient density; LND, low-nutrient density; HND+EO, HND diet added with $150 \mathrm{mg} / \mathrm{kg}$ of essential oils (EO); LND+EO, LND diet added with $150 \mathrm{mg} / \mathrm{kg}$ of EO; SEM, pooled standard error of the mean.

${ }^{1}$ Values $(\mathrm{g} / 100 \mathrm{~g}$ of body weight) are expressed as means of seven replicates per dietary group.

microflora (Table 4) and blood parameters such as total cholesterol, GOT, GPT, and ND- and IB-specific antibodies (Table 5).

\section{DISCUSSION}

The present study was designed to investigate whether dietary EO added into different nutrient density diets could improve growth performance of broiler chickens. Initially, a positive effect was expected in the light of previous reports showing that dietary EO increased growth performance and nutrient utilization in broiler chickens (Hernandez et al., 2004; Mountzouris et al., 2011; Bravo et al., 2014; Cho et al., 2014). In this study, we confirmed the previous reports (Buchanan et al., 2008; Leeson, 2012) on the wellestablished negative effect of nutrient density on growth performance (e.g., decrease in live body weight, feed intake, and daily weight gain) in broiler chickens. It was observed that EO supplementation, regardless of nutrient density, significantly increased $(\mathrm{p}<0.05)$ daily body weight gain during the periods of 22 to 35 and 1 to 35 days, and also improved feed conversion ratio during the periods of 22 to 35 and 1 to 35 days. The consistent increase in daily weight gain and decrease in feed conversion ratio by dietary EO would be likely the consequence of increased nutrient utilization as reported elsewhere (Hernandez et al., 2004; Lee et al., 2004; Mountzouris et al., 2011; Bravo et al., 2014; Cho et al., 2014). However, we did not measure nutrients digestibility or digestive enzyme activities in this study.

It is of note that EO-mediated increase in growth performance was equally effective in chickens fed diet containing different nutrient densities. In previous studies

Table 4. Effect of dietary essential oils on cecal microflora in broiler chickens fed diets containing different nutrient densities ${ }^{1}$

\begin{tabular}{lcccccccc}
\hline & \multirow{2}{*}{ HND } & \multirow{2}{*}{ LND } & \multirow{2}{*}{ HND+EO } & \multirow{2}{*}{ LND+EO } & \multirow{2}{*}{ Pooled SEM } & \multicolumn{3}{c}{$\mathrm{p}$-values } \\
\cline { 6 - 9 } & & & & & & Diet & EO & Diet $\times$ EO \\
\hline Total microbes (log cfu/g) & 6.25 & 6.59 & 6.59 & 6.65 & 0.228 & 0.818 & 0.491 & 0.927 \\
Lactic acid bacteria $(\log \mathrm{cfu} / \mathrm{g})$ & 6.56 & 6.76 & 7.04 & 6.61 & 0.228 & 0.644 & 0.413 & 0.608 \\
Coli forms (log cfu/g) & 6.01 & 5.39 & 5.63 & 5.92 & 0.241 & 0.742 & 0.328 & 0.566 \\
\hline
\end{tabular}

HND, high-nutrient density; LND, low-nutrient density; HND+EO, HND diet added with $150 \mathrm{mg} / \mathrm{kg}$ of essential oils (EO); LND+EO, LND diet added with $150 \mathrm{mg} / \mathrm{kg}$ of EO; SEM, pooled standard error of the mean.

${ }^{1}$ Values are expressed as means of seven replicates per dietary group.

Table 5. Effects of dietary essential oils on blood characteristics and antibody titers against Newcastle disease (ND) virus and infectious bronchitis (IB) in broiler chickens fed diets containing different nutrient densities ${ }^{1}$

\begin{tabular}{|c|c|c|c|c|c|c|c|c|}
\hline & \multirow{2}{*}{ HND } & \multirow{2}{*}{ LND } & \multirow{2}{*}{$\mathrm{HND}+\mathrm{EO}$} & \multirow{2}{*}{$\mathrm{LND}+\mathrm{EO}$} & \multirow{2}{*}{ Pooled SEM } & \multicolumn{3}{|c|}{ p-values } \\
\hline & & & & & & Diet & EO & Diet $\times \mathrm{EO}$ \\
\hline Total cholesterol (mg/dL) & 92.05 & 90.00 & 90.47 & 90.97 & 4.59 & 0.867 & 0.948 & 0.784 \\
\hline GOT (U/L) & 277.20 & 281.62 & 278.31 & 278.33 & 3.86 & 0.571 & 0.780 & 0.574 \\
\hline GPT (U/L) & 8.86 & 8.90 & 8.87 & 8.98 & 0.40 & 0.853 & 0.911 & 0.931 \\
\hline \multicolumn{9}{|l|}{ Viral antibody titer $\left(\log _{10}\right)$} \\
\hline ND titer & 2.29 & 2.00 & 2.57 & 2.43 & 0.300 & 0.480 & 0.248 & 0.805 \\
\hline IB titer & 3.00 & 4.00 & 3.00 & 3.29 & 0.392 & 0.113 & 0.375 & 0.375 \\
\hline
\end{tabular}

HND, high-nutrient density; LND, low-nutrient density; HND+EO, HND diet added with $150 \mathrm{mg} / \mathrm{kg}$ of essential oils (EO); LND+EO, LND diet added with $150 \mathrm{mg} / \mathrm{kg}$ of EO; SEM, pooled standard error of the mean; GOT, glutamic-oxaloacetic transaminase; GPT, glutamic-pyruvic transaminase.

${ }^{1}$ Values are expressed as means of seven replicates per dietary group. 
with EO supplemented into different nutrient density, the manuscript. conflicted results have been reported. For example, Buchanan et al. (2008) observed an increase in feed efficiency in broiler chickens fed the EO-added, HND diet, but this effect was not seen when the LND diet was used. Bozkurt et al. (2012) reported that body weights were increased in broiler chickens fed the EO-fortified, wheatbased diet, but were significantly decreased when EO was added into the corn-based diet. Finally, dietary EO did not improve overall growth performance in broiler chickens fed a threonine-deficient diet (Muhl and Liebert, 2007). In this study, the LND vs HND diet contained less energy and CP, but formulated to keep the ratios of lysine to limiting amino acids constant. Whether that the effect of dietary EO on growth performance is more effective in low energy/nutrient density diets with balanced amino acids needs to be addressed. Nonetheless, our study provides evidence that dietary EO could improve growth performance of broiler chicken and the EO-mediated effect was independent to nutrient density. The latter finding is considered of practical relevance in feed formulation in poultry production. According to Leeson (2012), the LND vs HND diet will be feasible in poultry production as the feed prices are currently on the increase.

None of parameters measured in this study were affected by either nutrient density or dietary EO. However, it was found that there were significant interactions $(p<0.05)$ between density and EO on the relative weight of bursa of Fabricius and abdominal fat. Dietary EO when added into a LND diet increased relative weight of bursa of Fabricius, which reached to the value shown in birds fed a HND diet alone. Whether this indicates the consequence of altered immune status by EO needs to be verified as no difference in humoral responses against ND and IB were observed in this study. To our surprise, addition of EO into the HND, but not LND diet, tended to increase the relative abdominal fat, thus leading to significant treatment interaction. At this stage, clear explanation on the confounding result is not readily available.

In conclusion, the present study showed that dietary EO, independent to nutrient density, effectively increased body weight, daily weight gains and feed conversion ratio in broiler chickens. In addition, there were significant treatment interactions on relative bursal of Fabricius and abdominal fats. Finally, none of parameters measured, i.e., relative organ weights, percentage of meat yields, cecal microflora, blood characteristics were affected by either nutrient density or dietary EO.

\section{CONFLICT OF INTEREST}

We certify that there is no conflict of interest with any financial organization regarding the material discussed in

\section{ACKNOWLEDGMENTS}

This paper was supported by the KU Research Professor Program of KonKuk University, and Delacon Biotechnik Ges.m.b.H., and also partially supported by the grant "Investigation of black rice extract on functional mechanism modulating bone health and obesity using in vivo method (PJ01009001)" of Rural Development Administration, Republic of Korea. We thank In-Sook An in the Poultry Science Laboratory, Department of Animal Science and Technology, College of Animal Bioscience and Technology, KonKuk University for the technical assistance.

\section{REFERENCES}

Bozkurt, M., K. Kucukyilmaz, A. U. Catli, Z. Ozyildiz, M. Cinar, M. Cabuk, and F. Coven. 2012. Influences of an essential oil mixture supplementation to corn versus wheat-based practical diets on growth, organ size, intestinal morphology and immune response of male and female broilers. Ital. J. Anim. Sci. 11:e54.

Bravo, D., V. Pirgozliev, and S. P. Rose. 2014. A mixture of carvacrol, cinnamaldehyde, and capsicum oleoresin improves energy utilization and growth performance of broiler chickens fed maize-based diet. J. Anim. Sci. 92:1531-1536.

Brickett, K. E., J. P. Dahiya, H. L. Classen, and S. Gomis. 2007. Influence of dietary nutrient density, feed form, and lighting on growth and meat yield of broiler chickens. Poult. Sci. 86:21722181.

Buchanan, N. P., J. M. Hott, S. E. Cutlip, A. L. Rack, A. Asamer, and J. S. Moritz. 2008. The effects of a natural antibiotic alternative and a natural growth promoter feed additive on broiler performance and carcass quality. J. Appl. Poult. Res. 17:202-210.

Cho, J. H., H. J. Kim, and I. H. Kim. 2014. Effects of phytogenic feed additive on growth performance, digestibility, blood metabolites, intestinal microbiota, meat color and relative organ weight after oral challenge with Clostridium perfringens in broilers. Livest. Sci. 160:82-88.

Hernandez, F., J. Madrid, V. Garcia, J. Orengo, and M. D. Megias. 2004. Influence of two plant extracts on broilers performance, digestibility, and digestive organ size. Poult. Sci. 83:169-174.

Huyghebaert, G., R. Ducatelle, and F. Van Immerseel. 2011. An update on alternatives to antimicrobial growth promoters for broilers. Vet. J. 187:182-188.

Jamroz, D., A. Wiliczkiewicz, T. Wertelecki, J. Orda, and J. Skorupinska. 2005. Use of active substances of plant origin in chicken diets based on maize and locally grown cereals. Br. Poult. Sci. 46:485-493.

Khattak, F., A. Ronchi, P. Castelli, and N. Sparks. 2014. Effects of natural blend of essential oil on growth performance, blood biochemistry, cecal morphology, and carcass quality of broiler chickens. Poult. Sci. 93:132-137.

Lee, K. W., H. Everts, and A. C. Beynen. 2004. Essential oils in broiler nutrition. Int. J. Poult. Sci. 3:738-752.

Lee, S. H., H. S. Lillehoj, S. I. Jang, K. W. Lee, M. S. Parks, D. Bravo, and E. P. Lillehoj. 2011. Cinnamaldehyde enhances in 
vitro parameters of immunity and reduces in vivo infection against avian coccidiosis. Br. J. Nutr. 106:862-869.

Lee, K. W., H. S. Lillehoj, S. I. Jang, and S. H. Lee. 2012. Effects of various field coccidiosis control programs on host innate and adaptive immunity in commercial broiler chickens. Korean J. Poult. Sci. 39:17-25.

Lee, D. W., J. H. Shin, J. M. Park, J. C. Song, H. J. Shu, U. J. Chang, B. K. An, C. W. Kang, and J. M. Kim. 2010. Growth performance and meat quality of broiler chicks fed germinated and fermented soybeans. Korean J. Food Sci. Anim. Resour. 30:938-945.

Leeson, S. 2012. Future considerations in poultry nutrition. Poult. Sci. 941:1281-1285.

Li, W. B., Y. L. Guo, J. L. Chen, R. Wang, Y. He, and D. G. Su. 2010. Influence of lighting schedule and nutrient density in broiler chickens: effect on growth performance, carcass traits and meat quality. Asian Australas. J. Anim. Sci. 23:1510-1518.

Mirshekar, R., B. Dastar, B. Shabanpour, and S. Hassani. 2013. Effect of dietary nutrient density and vitamin premix withdrawal on performance and meat quality of broiler chickens. J. Sci. Food Agric. 93:2979-2985.

Mountzouris, K. C., V. Paraskevas, P. Tsirtsikos, I. Palamidi, T. Steiner, G. Schatzmayr, and K. Fegeros. 2011. Assessment of a phytogenic feed additive effect on broiler growth performance, nutrient digestibility and caecal microflora composition. Anim. Feed Sci. Technol. 168:223-231.

Muhl, A. and F. Liebert. 2007. Growth, nutrient utilization and threonine requirement of growing chicken fed threonine limiting diets with commercial blends of phytogenic feed additives. J. Poult. Sci. 44:297-304
Noh, H. S, S. L. Ingale, S. H. Lee, K. H. Kim, I. K. Kwon, Y. H. Kim, and B. J. Chae. 2014. Effects of citrus pulp, fish byproduct and Bacillus subtilis fermentation biomass on growth performance, nutrient digestibility, and fecal microflora of weanling pigs. J. Anim. Sci. Technol. 56:10.

SAS. 2002. SAS User's Guide. Statistics, Version 8, SAS Institute Inc., Cary, NC, USA.

Scheuermann, G. N., A. C. Junior, L. Cyprioano, and A. M. Gabbi. 2009. Phytogenic additive as an alternative to growth promoters in broiler chickens. Cienc. Rural 39:522-527.

Yan, L., J. P. Wang, H. J. Kim, Q. W. Meng, X. Ao, S. M. Hong, and I. H. Kim. 2010. Influence of essential oil supplementation and diets with different nutrient densities on growth performance, nutrient digestibility, blood characteristics, meat quality and fecal noxious gas content in grower-finisher pigs. Livest. Sci. 128:115-122.

Zeng, Z. K., X. Xu, Q. Zhang, P. Li, P. Zhao, Q. Li, J. Liu, and X. Piao. 2015a. Effects of essential oil supplementation of a lowenergy diet on performance, intestinal morphology and microflora, immune properties and antioxidant activities in weaned pigs. Anim. Sci. J. 86:279-285.

Zeng, Z., S. Zhang, H. Wang, and X. Piao. 2015b. Essential oil and aromatic plants as feed additives in non-ruminant nutrition: A review. J. Anim. Sci. Biotechnol. 6:7.

Zhao, J. P., J. L. Chen, G. P. Zhao, M. Q. Zheng, R. R. Jiang, and J. Wen. 2009. Live performance, carcass composition, and blood metabolite responses to dietary nutrient density in two distinct broiler breeds of male chickens. Poult. Sci. 88:2575-2584. 